\title{
SOLVABLE POINTS ON SMOOTH PROJECTIVE VARIETIES
}

\author{
TREVOR D. WOOLEY
}

\begin{abstract}
We establish that smooth, geometrically integral projective varieties of small degree are not pointless in suitable solvable extensions of their field of definition, provided that this field is algebraic over $\mathbb{Q}$.
\end{abstract}

\section{INTRODUCTION}

Given a field $K$ of characteristic 0 , consider the compositum $K^{\text {sol }}$ of all solvable extensions of $K$. It was shown by Abel in 1823 that polynomials of degree 5 or more in a single variable need not have their roots defined over $K^{\text {sol }}$. There has been recent speculation that perhaps $K^{\text {sol }}$ is so large that any geometrically irreducible projective curve defined over $K$ should possess a point defined in $K^{\text {sol }}$ (see, for example, Pál [12, Question 1.2] and Wooley [17, page 63]). Such has been confirmed by Pál [12, Theorem 1.6] for smooth curves of genus $0,2,3$ and 4. Much progress has also been made towards confirmation of this conjecture for curves of genus 1 in the case $K=\mathbb{Q}$ by Çiperiani and Wiles [5]. The situation, however, remains unclear both for curves of higher genus and higher dimensional varieties. On the one hand, Pál [12, Theorem 1.5] has shown that when $g \geqslant 40$, there are local fields $F$ for which there exists a curve of genus $g$ failing to possess any point defined over $F^{\text {sol }}$. On the other hand, Pál [12, Theorem 1.7] has proved that any smooth, geometrically rational projective surface possesses a point defined over a solvable extension of its field of definition. In the absence of a more definitive resolution of this conjecture concerning solvable points on curves, and its analogue for surfaces, one is naturally led to enquire whether varieties of larger dimension might be guaranteed to possess solvable points. In this note, we establish that smooth, geometrically integral projective varieties are not pointless in solvable extensions of their field of definition, assumed algebraic over $\mathbb{Q}$, whenever their dimension is large enough in terms of their degree.

Our conclusions are in principle rather more general than the previous paragraph might suggest, though this observation hinges on the Lefschetz principle. It suffices here to describe the latter as asserting that any reasonable statement in algebraic geometry true over $\mathbb{C}$ is also true over any algebraically closed field of characteristic 0 . It seems fair to comment that there remains considerable uncertainty concerning the extent to which such a statement is true, or indeed makes sense (see Eklof [7] and Seidenberg [13]). Thus, with safety in mind, we 
will restrict our conclusions to algebraic extensions of $\mathbb{Q}$, noting the potential for extension to arbitrary fields of characteristic 0 .

Theorem 1.1. Let $X \subseteq \mathbb{P}^{n}$ be a smooth and geometrically integral variety defined over a field $K$ algebraic over $\mathbb{Q}$. Then $X$ possesses a point defined over a solvable extension of $K$ provided only that $\operatorname{dim}(X) \geqslant 2^{2^{\operatorname{deg}(X)}}$.

The lower bound constraint on the dimension is certainly large, and it is worth noting that improvement is certainly possible, especially for smaller degrees. However, when $\operatorname{deg}(X)$ is large, it seems that our methods are incapable of reducing this constraint in Theorem 1.1 to one of the shape $\operatorname{dim}(X) \geqslant 2^{2^{c \operatorname{deg}(X)}}$, for any $c<1$.

Our strategy for proving this theorem is simple. As has recently been observed by Browning and Heath-Brown [4], it follows from work of Bertram, Ein and Lazarsfeld [1] that a (complex) smooth variety of dimension large enough in terms of its degree is automatically a complete intersection, and moreover its annihilating ideal is generated by forms defined over its field of definition. Having modified this strategy to the context of $K^{\text {sol }}$ in $\S 2$, we apply a diagonalisation method in $\S 3$, based on that due to Brauer [2], to show that this complete intersection has a point defined in a solvable extension of the groundfield. The key input from the solubility of diagonal equations here is the trivial observation that, when $a_{0}, a_{1} \in K^{\times}$, then the diagonal equation $a_{0} x_{0}^{d}+a_{1} x_{1}^{d}=0$ possesses a solution in which $x_{0}$ and $x_{1}$ both lie in a solvable extension of $K$, namely $K\left(\sqrt[d]{-a_{1} / a_{0}}\right)$. It follows, in fact, that the point lying on $X$ derived in Theorem 1.1 lies in a solvable field extension of $K$ defined by taking a tower of field extensions, each of degree no larger than $\operatorname{deg}(X)$. The elementary nature of our argument ensures that generalisations are easily obtained, and we mention a few in $\S 4$.

\section{Passage to a COMplete intersection}

We begin by adapting the treatment of Browning and Heath-Brown [4] so that the groundfield is no longer restricted to be $\mathbb{Q}$. Throughout this section, we assume $K$ to be an algebraic extension of $\mathbb{Q}$. Let $X \subseteq \mathbb{P}^{n}$ be a smooth and geometrically integral variety defined over $K$. From Harris [9, Corollary 18.12], this variety lies in a linear subspace of dimension at most $\operatorname{dim}(X)+\operatorname{deg}(X)-1$. By considering the action of $\mathrm{Gal}(\bar{K}: K)$ on this linear space, it is apparent that there is no loss of generality in supposing it to be defined over $K$. We may therefore suppose without loss that

$$
n+1 \leqslant \operatorname{dim}(X)+\operatorname{deg}(X) .
$$

Next, Bertram, Ein and Lazarsfeld [1, Corollary 3] show that whenever $X \subseteq \mathbb{P}^{n}$ is smooth and $\operatorname{deg}(X) \leqslant \frac{1}{2} n /(n-\operatorname{dim}(X))$, then $X$ is a complete intersection. Then, as in [4, §1], we deduce from (2.1) that such is the case whenever

$$
\operatorname{dim}(X)>\operatorname{deg}(X)(2 \operatorname{deg}(X)-3) .
$$

The argument of [4, Lemma 3.3] adapts to give the following conclusion. 
Lemma 2.1. Let $X \subseteq \mathbb{P}^{n}$ be a smooth complete intersection of codimension $R$ which is globally defined over $K$. Then there exist forms $F_{1}, \ldots, F_{R} \in$ $K\left[x_{0}, \ldots, x_{n}\right]$ such that the annihilating ideal of $X$ is generated by $\left\{F_{1}, \ldots, F_{R}\right\}$.

Proof. This conclusion is immediate from [4, Lemma 3.3] in the case $K=\mathbb{Q}$, and the argument of its proof applies mutatis mutandis to deliver the more general conclusion recorded here. In essence, one applies the action of $\operatorname{Gal}(\bar{K}: K)$ to push the field of definition of the coefficients of elements of the annihilating ideal of $X$ down to the ground field by applying the natural trace operator.

Finally, we record an immediate generalisation of a lemma presented in [4].

Lemma 2.2. Let $\left\{F_{1}, \ldots, F_{R}\right\} \subseteq K\left[x_{0}, \ldots, x_{n}\right]$ be a a non-singular system of forms defining a variety $X$ in $\mathbb{P}^{n}$. Then the annihilating ideal of $X$ is generated by $\left\{F_{1}, \ldots, F_{R}\right\}$, and $X$ is a smooth complete intersection of codimension $R$. In addition, the variety $X$ is geometrically integral, and has degree

$$
\operatorname{deg}(X)=\operatorname{deg}\left(F_{1}\right) \cdots \operatorname{deg}\left(F_{R}\right) .
$$

Proof. The desired conclusion is established in [4, Lemma 3.2] when the ground field is $\mathbb{Q}$. The argument of the latter proof applies, mutatis mutandis, in the present setting.

We are now equipped to derive the principal conclusion of this section.

Lemma 2.3. Let $X \subseteq \mathbb{P}^{n}$ be a smooth and geometrically integral variety, defined over a field $K$ algebraic over $\mathbb{Q}$, and satisfying the condition

$$
\operatorname{dim}(X)>2 \operatorname{deg}(X)(2 \operatorname{deg}(X)-3) .
$$

Then there exist forms $F_{1}, \ldots, F_{R} \in K\left[x_{0}, \ldots, x_{n}\right]$ satisfying the conditions:

(a) $R=n-\operatorname{dim}(X)$;

(b) $\operatorname{deg}(X)=\operatorname{deg}\left(F_{1}\right) \cdots \operatorname{deg}\left(F_{R}\right)$;

(c) the point $\left(y_{0}: y_{1}: \ldots: y_{n}\right) \in \mathbb{P}^{n}$ lies on $X$ if and only if

$$
F_{j}\left(y_{0}, \ldots, y_{n}\right)=0 \quad(1 \leqslant j \leqslant R) .
$$

Proof. Under the hypothesis (2.2), it follows from Lemma 2.1 and its preamble that with $R=n-\operatorname{dim}(X)$, there exist forms $F_{1}, \ldots, F_{R} \in K\left[x_{0}, \ldots, x_{n}\right]$ such that the annihilating ideal of $X$ is generated by $\left\{F_{1}, \ldots, F_{R}\right\}$. The claim (c) is an immediate consequence of the latter conclusion. Moreover, since $\left\{F_{1}, \ldots, F_{R}\right\}$ must be a non-singular system of forms, it follows from Lemma 2.2 that $\operatorname{deg}(X)=\operatorname{deg}\left(F_{1}\right) \cdots \operatorname{deg}\left(F_{R}\right)$. This completes the proof of the lemma.

\section{BRAUER DiAgONALISATION}

We examine the existence of rational points on the complete intersection emerging from the previous section by means of a variant of the diagonalisation argument employed by Brauer [2] in his work on Hilbert's resolvant problem. Let $\mathbb{K}$ be a field. Denote by $\mathcal{G}_{d}^{(m)}\left(r_{d}, \ldots, r_{1}\right)$ the set of $\left(r_{d}+\ldots+r_{1}\right)$-tuples of homogeneous polynomials, of which $r_{i}$ have degree $i$ for $1 \leqslant i \leqslant d$, with 
coefficients in $\mathbb{K}$, possessing no non-trivial linear space of $\mathbb{K}$-rational solutions of projective dimension $m$. Define $V_{d}^{(m)}(\mathbf{r})=V_{d}^{(m)}\left(r_{d}, \ldots, r_{1} ; \mathbb{K}\right)$ by putting

$$
V_{d}^{(m)}\left(r_{d}, \ldots, r_{1} ; \mathbb{K}\right)=\sup _{\mathbf{h} \in \mathcal{G}_{d}^{(m)}\left(r_{d}, \ldots, r_{1}\right)} \nu(\mathbf{h}),
$$

in which $\nu(\mathbf{h})$ denotes the number of variables appearing explicitly in $\mathbf{h}$. Likewise, denote by $\mathcal{D}_{d, r}$ the set of $r$-tuples of diagonal polynomials of degree $d$, with coefficients in $\mathbb{K}$, which possess no non-trivial zeros over $\mathbb{K}$, and put

$$
\phi_{d, r}(\mathbb{K})=\sup _{\mathbf{f} \in \mathcal{D}_{d, r}} \nu(\mathbf{f}) .
$$

Note that $V_{d}^{(m)}\left(r_{d}, \ldots, r_{1} ; \mathbb{K}\right)$ is an increasing function of the arguments $m$ and $r_{d}, \ldots, r_{1}$. We abbreviate $V_{d}^{(0)}(\mathbf{r} ; \mathbb{K})$ to $V_{d}(\mathbf{r} ; \mathbb{K})$, and $V_{d}(r, 0, \ldots, 0 ; \mathbb{K})$ to $v_{d, r}(\mathbb{K})$. In additon, we abbreviate $\phi_{d, 1}(\mathbb{K})$ to $\phi_{d}(\mathbb{K})$, and put

$$
\psi_{d}(\mathbb{K})=\sup _{1 \leqslant i \leqslant d} \phi_{i}(\mathbb{K}) .
$$

We drop mention of $\mathbb{K}$ from all of these notations when the field of definition $\mathbb{K}$ is fixed. Note that whenever $n>\phi_{d}(\mathbb{K})$, and $a_{i} \in \mathbb{K}(0 \leqslant i \leqslant n)$, then the equation $a_{0} x_{0}^{d}+\ldots+a_{n} x_{n}^{d}=0$ has a non-trivial solution over $\mathbb{K}$. A similar conclusion applies, concerning the existence of non-trivial linear spaces of solutions, regarding the notation $V_{d}^{(m)}\left(r_{d}, \ldots, r_{1} ; \mathbb{K}\right)$.

We first record [11, equation (3.1)] in the form embodied in [16, Lemma 2.3].

Lemma 3.1. When $m$ is a positive integer, one has

$$
V_{d}^{(m)}\left(r_{d}, \ldots, r_{1} ; \mathbb{K}\right) \leqslant m+V_{d}\left(t_{d}, \ldots, t_{1} ; \mathbb{K}\right)
$$

where

$$
t_{j}=\sum_{i=j}^{d} r_{i} m^{i-j} \quad(1 \leqslant j \leqslant d) .
$$

Recall next the efficient diagonalisation procedure given in [16, Lemma 2.2].

Lemma 3.2. Let $d$ and $r_{i}(1 \leqslant i \leqslant d)$ be non-negative integers with $d \geqslant 2$ and $r_{d}>0$. Then whenever $\phi_{d}<\infty$ one has

$$
V_{d}\left(r_{d}, \ldots, r_{1} ; \mathbb{K}\right) \leqslant r_{d} \phi_{d}+V_{d-1}\left(s_{d-1}, \ldots, s_{1} ; \mathbb{K}\right),
$$

where

$$
s_{j}=\sum_{i=j}^{d} r_{i}\left(r_{d} \phi_{d}\right)^{i-j} \quad(1 \leqslant j \leqslant d-1) .
$$

We ultimately apply Lemmata 3.1 and 3.2 only in situations wherein the arguments $r_{i}$ are distributed in a certain restricted manner. In order to facilitate the announcement of our key lemma, we describe a $d$-tuple $\left(r_{d}, \ldots, r_{1}\right)$ as being solid when $r_{i}^{2} \geqslant r_{i-1}$ for $1<i \leqslant d$. 
Lemma 3.3. Let $d$ and $r_{d}, \ldots, r_{1}$ be positive integers with $d \geqslant 2$ satisfying the property that $\left(r_{d}, \ldots, r_{1}\right)$ is solid. Then provided that $\psi_{d}(\mathbb{K})<\infty$, one has

$$
V_{d}\left(r_{d}, \ldots, r_{1} ; \mathbb{K}\right) \leqslant 2 r_{d}^{2^{d-1}}\left(\psi_{d}+1\right)^{2^{d-1}-1} .
$$

Proof. It follows from Lemma 3.2 that

$$
V_{d}\left(r_{d}, \ldots, r_{1} ; \mathbb{K}\right) \leqslant V_{d-1}\left(s_{d-1}, \ldots, s_{2}, s_{1}+r_{d} \phi_{d} ; \mathbb{K}\right),
$$

where

$$
s_{j}=\sum_{i=j}^{d} r_{i}\left(r_{d} \phi_{d}\right)^{i-j} \quad(1 \leqslant j \leqslant d-1) .
$$

Observe that for every integer $u$, one has $u \leqslant 2^{u-1}$. Furthermore, when $u$ and $v$ are non-negative integers with $u \leqslant v$, one has $v-u \leqslant 2^{v}-2^{u}$. Thus, the hypothesis that $\left(r_{d}, \ldots, r_{1}\right)$ is solid implies that for $1<j \leqslant d-1$, one has

$$
s_{j} \leqslant \sum_{u=0}^{d-j} r_{d}^{2^{u}}\left(r_{d} \phi_{d}\right)^{d-j-u} \leqslant \sum_{u=0}^{d-j} r_{d}^{2^{u}} r_{d}^{2^{d-j}-2^{u}} \phi_{d}^{d-j-u}
$$

whence

$$
s_{j} \leqslant r_{d}^{2^{d-j}}\left(\phi_{d}+1\right)^{d-j} \leqslant r_{d}^{2^{d-j}}\left(\phi_{d}+1\right)^{2^{d-j-1}} .
$$

Moreover, one sees in like manner that when $d \geqslant 3$, then

$$
s_{1}+r_{d} \phi_{d} \leqslant r_{d} \phi_{d}+r_{d}^{2^{d-1}} \sum_{u=0}^{d-1} \phi_{d}^{d-1-u} \leqslant r_{d}^{2^{d-1}} \sum_{u=0}^{d-1}\left(\begin{array}{c}
d-1 \\
u
\end{array}\right) \phi_{d}^{d-1-u},
$$

so that

$$
s_{1}+r_{d} \phi_{d} \leqslant r_{d}^{2^{d-1}}\left(\phi_{d}+1\right)^{d-1} \leqslant r_{d}^{2^{d-1}}\left(\phi_{d}+1\right)^{2^{d-2}},
$$

whilst, in the situation with $d=2$, one has

$$
s_{1}+r_{d} \phi_{d} \leqslant r_{d} \phi_{d}+r_{d}^{2^{d-1}}\left(\phi_{d}+1\right) \leqslant 2 r_{d}^{2^{d-1}}\left(\phi_{d}+1\right)^{2^{d-2}} .
$$

Consequently, whenever $d \geqslant 2$ and $\left(r_{d}, \ldots, r_{1}\right)$ is solid, then

$$
V_{d}\left(r_{d}, \ldots, r_{1} ; \mathbb{K}\right) \leqslant V_{d-1}\left(\omega_{d-1}, \omega_{d-1}^{2}, \ldots, \omega_{d-1}^{2^{d-3}}, \delta \omega_{d-1}^{2^{d-2}} ; \mathbb{K}\right),
$$

where $\omega_{d-1}=r_{d}^{2}\left(\phi_{d}+1\right)$ and

$$
\delta= \begin{cases}1, & \text { when } d \geqslant 3 \\ 2, & \text { when } d=2\end{cases}
$$

The relation (3.1) may be applied inductively to show that for each integer $u$ with $1 \leqslant u \leqslant d-2$, one has

$$
V_{d}\left(r_{d}, \ldots, r_{1} ; \mathbb{K}\right) \leqslant V_{d-u}\left(\omega_{d-u}, \omega_{d-u}^{2}, \ldots, \omega_{d-u}^{2^{d-u-1}} ; \mathbb{K}\right),
$$

where for each $j$ we write

$$
\omega_{j}=r_{d}^{2^{d-j}}\left(\psi_{d}+1\right)^{2^{d-j}-1} .
$$


This claimed relation follows from (3.1) when $u=1$, providing the base of the induction. Let $U$ be an integer with $2 \leqslant U \leqslant d-2$, and assume that (3.2) holds for $1 \leqslant u<U$. Then we find from (3.1) that

$$
\begin{aligned}
V_{d}\left(r_{d}, \ldots, r_{1} ; \mathbb{K}\right) & \leqslant V_{d-U+1}\left(\omega_{d-U+1}, \omega_{d-U+1}^{2}, \ldots, \omega_{d-U+1}^{2^{d-U}} ; \mathbb{K}\right) \\
& \leqslant V_{d-U}\left(\Omega, \Omega^{2}, \ldots, \Omega^{2^{d-U+1}} ; \mathbb{K}\right)
\end{aligned}
$$

where

$$
\Omega=\omega_{d-U+1}^{2}\left(\phi_{d-U+1}+1\right) \leqslant\left(r_{d}^{2^{U-1}}\left(\psi_{d}+1\right)^{2^{U-1}-1}\right)^{2}\left(\psi_{d}+1\right)=\omega_{d-U} .
$$

This confirms the inductive step, so that, in particular, one has

$$
V_{d}\left(r_{d}, \ldots, r_{1} ; \mathbb{K}\right) \leqslant V_{2}\left(\omega_{2}, \omega_{2}^{2} ; \mathbb{K}\right) .
$$

From here, an additional application of (3.1) delivers the bound

$$
V_{d}\left(r_{d}, \ldots, r_{1} ; \mathbb{K}\right) \leqslant V_{1}\left(2 \omega_{1} ; \mathbb{K}\right)=2 \omega_{1}=2 r_{d}^{2^{d-1}}\left(\psi_{d}+1\right)^{2^{d-1}-1} .
$$

This completes the proof of the lemma.

We refine Lemma 3.3 when all implicit equations have the same degree.

Lemma 3.4. Let $d$ and $r$ be positive integers with $d \geqslant 2$. Then whenever $\psi_{d}(\mathbb{K})<\infty$, one has

$$
v_{d, r}(\mathbb{K}) \leqslant r \phi_{d}+2\left(r^{2} \phi_{d}\right)^{2^{d-2}}\left(\psi_{d-1}+1\right)^{2^{d-2}-1} .
$$

Proof. It follows from Lemma 3.2 that

$$
v_{d, r}(\mathbb{K}) \leqslant r \phi_{d}+V_{d-1}\left(s_{d-1}, \ldots, s_{1} ; \mathbb{K}\right),
$$

where $s_{j}=r\left(r \phi_{d}\right)^{d-j}(1 \leqslant j \leqslant d-1)$. We therefore find that

$$
s_{j} \leqslant\left(r^{2} \phi_{d}\right)^{2^{d-j-1}} \quad(1 \leqslant j \leqslant d-1) .
$$

But $\left(s_{d-1}, \ldots, s_{1}\right)$ is solid, and hence Lemma 3.3 delivers the bound

$$
V_{d-1}\left(s_{d-1}, \ldots, s_{1} ; \mathbb{K}\right) \leqslant 2 s_{d-1}^{2^{d-2}}\left(\psi_{d-1}+1\right)^{2^{d-2}-1} .
$$

The conclusion of the lemma is now immediate from (3.3).

Finally, we combine Lemmata 3.1 and 3.3 to provide a conclusion of use in investigating the existence of linear spaces of solutions.

Lemma 3.5. Let $d$ and $r_{d}, \ldots, r_{1}$ be positive integers with $d \geqslant 2$ satisfying the property that $\left(r_{d}, \ldots, r_{1}\right)$ is solid. Then provided that $\psi_{d}(\mathbb{K})<\infty$, one has

$$
V_{d}^{(m)}\left(r_{d}, \ldots, r_{1} ; \mathbb{K}\right) \leqslant m+2\left(r_{d}^{2}(m+1)\right)^{2^{d-2}}\left(\psi_{d}+1\right)^{2^{d-1}-1} .
$$

Proof. It follows from Lemma 3.1 that

$$
V_{d}^{(m)}\left(r_{d}, \ldots, r_{1} ; \mathbb{K}\right) \leqslant m+V_{d}\left(t_{d}, \ldots, t_{1} ; \mathbb{K}\right),
$$

where

$$
t_{j}=\sum_{i=j}^{d} r_{i} m^{i-j} \quad(1 \leqslant j \leqslant d) .
$$


The hypothesis that $\left(r_{d}, \ldots, r_{1}\right)$ is solid implies that for $1 \leqslant j \leqslant d$, one has

$$
t_{j} \leqslant \sum_{u=0}^{d-j} r_{d}^{2^{u}} m^{d-j-u} \leqslant r_{d}^{2^{d-j}}(m+1)^{d-j} \leqslant\left(r_{d}^{2}(m+1)\right)^{2^{d-j-1}} .
$$

The $d$-tuple $\left(t_{d}, \ldots, t_{1}\right)$ is solid, and hence Lemma 3.3 shows that

$$
V_{d}\left(t_{d}, \ldots, t_{1} ; \mathbb{K}\right) \leqslant 2 t_{d}^{2^{d-1}}\left(\psi_{d}+1\right)^{2^{d-1}-1} \leqslant 2\left(r_{d}^{2}(m+1)\right)^{2^{d-2}}\left(\psi_{d}+1\right)^{2^{d-1}-1} .
$$

The conclusion of the lemma now follows from (3.4).

\section{The Proof of Theorem 1.1, And Related CONCLUSions}

Theorem 1.1 follows from the case $m=0$ of the following theorem.

Theorem 4.1. Let $X \subseteq \mathbb{P}^{n}$ be a smooth and geometrically integral variety defined over a field $K$ algebraic over $\mathbb{Q}$. Then $X$ possesses a projective linear space of dimension $m$ defined over a solvable extension of $K$ provided only that $\operatorname{dim}(X) \geqslant(2(m+1))^{2^{\operatorname{deg}(X)}}$.

Proof. We begin with a straightforward consequence of Lemma 3.5. Let $K$ be a field algebraic over $\mathbb{Q}$, and consider a natural number $j$. Given any elements $a_{0}, a_{1} \in K^{\mathrm{sol}}$, the equation $a_{0} x_{0}^{j}+a_{1} x_{1}^{j}=0$ possesses the non-trivial solution $\left(x_{0}, x_{1}\right) \in\left(\sqrt[j]{a_{1}}, \sqrt[j]{-a_{0}}\right) \in K^{\mathrm{sol}} \times K^{\mathrm{sol}}$, and thus $\phi_{j}\left(K^{\mathrm{sol}}\right)=1$. It follows that $\psi_{d}\left(K^{\mathrm{sol}}\right)=1$ for every natural number $d$, and hence we deduce from Lemma 3.5 that whenever $d \geqslant 2$, and $\left(r_{d}, \ldots, r_{1}\right)$ is solid, then

$$
V_{d}^{(m)}\left(r_{d}, \ldots, r_{1} ; K^{\mathrm{sol}}\right) \leqslant\left(r_{d}^{2}(m+1)\right)^{2^{d-2}} 2^{2^{d-1}}+m .
$$

Next, let $X \subseteq \mathbb{P}^{n}$ be a smooth and geometrically integral variety defined over $K$. Let $m$ be a non-negative integer, and put $N=\operatorname{dim}(X)$ and $D=\operatorname{deg}(X)$. Suppose that $N \geqslant(2(m+1))^{2^{D}}$. Then it follows from Lemma 2.3 that for some positive integer $R$, there exist forms $F_{1}, \ldots, F_{R} \in K\left[x_{0}, \ldots, x_{n}\right]$, with respective degrees $d_{1}, \ldots, d_{R}$, satisfying the property that $\left(y_{0}: \ldots: y_{n}\right) \in \mathbb{P}^{n}$ is a $K^{\text {sol }}$-rational point on $X$ if and only if $F_{j}\left(y_{0}, \ldots, y_{n}\right)=0(1 \leqslant j \leqslant R)$. Moreover, we may suppose that

$$
n-R \geqslant(2(m+1))^{2^{D}} \quad \text { and } \quad D=d_{1} \cdots d_{R} .
$$

For $1 \leqslant j \leqslant D$, put $r_{j}=\operatorname{card}\left\{1 \leqslant i \leqslant R: d_{i}=j\right\}$. Then provided that $n \geqslant V_{D}^{(m)}\left(r_{D}, \ldots, r_{1} ; K^{\mathrm{sol}}\right)$, we see that $X$ possesses a $K^{\text {sol }}$-rational point. The conclusion of the theorem therefore follows on confirming that

$$
V_{D}^{(m)}\left(r_{D}, \ldots, r_{1} ; K^{\text {sol }}\right) \leqslant R+(2(m+1))^{2^{D}} .
$$

We divide into three cases, that in which $r_{1}=R$, a second in which $r_{1}<R$ and $r_{D} \geqslant 1$, and the final case with $r_{1}<R$ and $r_{D}=0$.

Suppose first that $r_{1}=R$, in which case $\left(r_{D}, \ldots, r_{1}\right)=(0, \ldots, 0, R)$. The trivial relation $V_{D}^{(m)}\left(0, \ldots, 0, R ; K^{\mathrm{sol}}\right)=R+m$, that is a consequence of linear algebra, then delivers (4.2) at once. 
Next, when $r_{1}<R$ and $r_{D} \geqslant 1$, it follows from the relation $D=d_{1} \cdots d_{R}$ that $\left(r_{d}, \ldots, r_{1}\right)$ takes the shape $\left(1,0, \ldots, 0, r_{1}\right)$ with $d \geqslant 2$. In such circumstances, one finds from (4.1) that

$$
\begin{aligned}
V_{D}^{(m)}\left(r_{D}, \ldots, r_{1} ; K^{\mathrm{sol}}\right) & \leqslant r_{1}+V_{D}^{(m)}\left(1,0, \ldots, 0 ; K^{\mathrm{sol}}\right) \\
& \leqslant R+(m+1)^{2^{D-2}} 2^{2^{D-1}}+m,
\end{aligned}
$$

and the desired upper bound (4.2) again follows.

Finally, suppose that $r_{1}<R$ and $r_{D}=0$. Here, sharper bounds than (4.2) are in fact available, though we are challenged by issues of complexity. Let

$$
d=\max \left\{1 \leqslant j \leqslant D: r_{j}>0\right\} \quad \text { and } \quad r=\max \left\{r_{j}: 2 \leqslant j \leqslant D\right\} .
$$

Then the relation $D=d_{1} \cdots d_{R}$ ensures that

$$
2 \leqslant d \leqslant \min \left\{D / 2, D / 2^{r-1}\right\} \quad \text { and } \quad 2^{r} \leqslant D .
$$

We now find from (4.1) that

$$
\begin{aligned}
V_{d}^{(m)}\left(r_{d}, \ldots, r_{1} ; K^{\mathrm{sol}}\right) & \leqslant r_{1}+V_{d}^{(m)}\left(r, r, \ldots, r ; K^{\mathrm{sol}}\right) \\
& \leqslant R+\left(r^{2}(m+1)\right)^{2^{d-2}} 2^{2^{d-1}}+m .
\end{aligned}
$$

But

$$
r^{2^{d-1}} \leqslant 2^{2^{d-1} r} \leqslant 2^{2^{d-1} \cdot 2^{r-1}} \leqslant 2^{2^{\nu-1}}
$$

where

$$
\nu=r+d-1 \leqslant r-1+\min \left\{D / 2, D / 2^{r-1}\right\} .
$$

One has $u+D / 2^{u} \geqslant u+1+D / 2^{u+1}$ whenever $D \geqslant 2^{u+1}$, so that since $2^{r} \leqslant D$, we discern that $\nu \leqslant 1+D / 2$. On substituting this bound into (4.4), and thence into (4.3), we deduce that

$$
\begin{aligned}
V_{d}^{(m)}\left(r_{d}, \ldots, r_{1} ; K^{\mathrm{sol}}\right) & \leqslant R+(m+1)^{2^{D-2}} 2^{2^{D / 2}} \cdot 2^{2^{D / 2-1}}+m \\
& \leqslant R+(m+1)^{2^{D}} 2^{2^{D}-1}+m .
\end{aligned}
$$

The desired bound (4.2) consequently follows in this final case.

Having confirmed the bound (4.2) in all cases, we conclude that $X$ contains

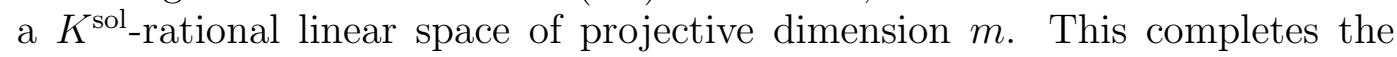
proof of the theorem, and hence also of Theorem 1.1.

We mention in passing two further conclusions that may be proved in a manner very similar to the proof of Theorem 4.1 .

Theorem 4.2. Suppose that $p$ is a rational prime, and let $X \subseteq \mathbb{P}^{n}$ be a smooth and geometrically integral variety defined over $\mathbb{Q}$. Then $X$ possesses a point defined over $\mathbb{Q}_{p}$ provided only that $\operatorname{dim}(X) \geqslant \operatorname{deg}(X)^{2^{\operatorname{deg}(X)}}$.

Proof. It follows from Davenport and Lewis [6, Theorem 1] that $\phi_{d}\left(\mathbb{Q}_{p}\right) \leqslant d^{2}$ for each natural number $d$. With $X \subseteq \mathbb{P}^{n}$ satisfying the hypotheses of the statement of the theorem, we put $N=\operatorname{dim}(X)$ and $D=\operatorname{deg}(X)$. Suppose that $N \geqslant D^{2^{D}}$. Then, as in the proof of Theorem 4.1, it follows that $X$ is a 
complete intersection defined over $\mathbb{Q}$, and further that the conclusion of the theorem follows provided we are able to establish the bound

$$
V_{D}\left(r_{D}, \ldots, r_{1} ; \mathbb{Q}_{p}\right) \leqslant R+D^{2^{D}},
$$

for all $D$-tuples $\left(r_{D}, \ldots, r_{1}\right)$ with $R=r_{D}+\ldots+r_{1}$ satisfying

$$
D=D^{r_{D}}(D-1)^{r_{D-1}} \cdots 2^{r_{2}} \text {. }
$$

When $r_{1}=R$, the bound (4.5) follows via linear algebra. Also, when $r_{1}<R$ and $r_{D} \geqslant 1$, one finds as before that $\left(r_{D}, \ldots, r_{1}\right)=\left(1,0, \ldots, 0, r_{1}\right)$. In such circumstances, an application of Lemma 3.4 gives

$$
\begin{aligned}
V_{D}\left(r_{D}, \ldots, r_{1} ; \mathbb{Q}_{p}\right) & \leqslant r_{1}+D^{2}+2\left(D^{2}\right)^{2^{D-2}}\left((D-1)^{2}+1\right)^{2^{D-2}-1} \\
& \leqslant R+2 D^{2^{D}-1} \leqslant R+D^{2^{D}}
\end{aligned}
$$

confirming (4.5). Finally, when $r_{1}<R$ and $r_{D}=0$, a treatment akin to that applied in the proof of Theorem 4.1 conveys us from Lemma 3.3 to the bound

$$
V_{D}\left(r_{D}, \ldots, r_{1} ; \mathbb{Q}_{p}\right) \leqslant R+D^{2^{D / 2+1}-1} \leqslant R+D^{2^{D}}
$$

again confirming (4.5). Thus $X$ does indeed possess a $\mathbb{Q}_{p}$-rational point. This completes the proof of the theorem.

Corollary 4.3. Let $X \subseteq \mathbb{P}^{n}$ be a smooth and geometrically integral variety defined over $\mathbb{Q}$. Then $X$ possesses a point defined over $\mathbb{Q}$ provided only that it possesses a real point and $\operatorname{dim}(X) \geqslant \operatorname{deg}(X)^{2^{\operatorname{deg}(X)}}$. In particular, when $\operatorname{deg}(X)$ is odd and the latter condition on the dimension is satisfied, then $X$ possesses a point defined over $\mathbb{Q}$.

Proof. It follows from Browning and Heath-Brown [4, Theorem 1.1] that $X$ satisfies the Hasse Principle provided only that $\operatorname{dim}(X) \geqslant(\operatorname{deg}(X)-1) 2^{\operatorname{deg}(X)}-1$. Since $d^{2^{d}}>(d-1) 2^{d}-1$ for $d \geqslant 1$, the first conclusion is immediate from Theorem 4.2. When $\operatorname{deg}(X)$ is odd, moreover, it follows from Lemma 2.3.(b) that $X$ is a complete intersection of hypersurfaces of odd degree. In such circumstances, it follows that $X$ possesses a real point (this follows as a consequence of the Borsuk-Ulam Theorem, or see as an alternative [10, Theorem 15]), and hence the desired result follows from the first conclusion of the theorem.

Theorem 4.4. Suppose that $p$ is a rational prime, and that $K$ is an algebraic extension of $\mathbb{Q}_{p}$. Let $L$ be an algebraic extension of $\mathbb{Q}$ embedding into $K$, and let $X \subseteq \mathbb{P}^{n}$ be a smooth and geometrically integral variety defined over $L$. Then $X$ possesses a point defined over $K$ provided only that

$$
\operatorname{dim}(X) \geqslant \exp \left(2^{\operatorname{deg}(X)+2}(\log \operatorname{deg}(X))^{2}\right) .
$$

Proof. The annihilating ideal of $X$ is defined by polynomials having coefficients in some finite field extension $L_{0}$ of $\mathbb{Q}$. It follows from Brink, Godinho and Rodrigues [3, Theorem 1] that when $d=p^{\tau} m$ with $p \nmid m$, and $K_{0}$ is any field extension of $\mathbb{Q}_{p}$ of finite degree, then one has $\phi_{d}\left(K_{0}\right) \leqslant d^{2 \tau+5}$. It follows that $\phi_{d}(K) \leqslant d^{2 \tau+5}$. The former conclusion improves on an earlier result of Skinner [15] (correcting [14]). Observe here that $\tau \leqslant(\log d) /(\log 2)$. A modicum of 
computation reveals that when $d \geqslant 3$, one has $2[(\log d) /(\log 2)]+5<8 \log d$, and thus $\phi_{d}(K)<\exp \left(8(\log d)^{2}\right)$. Note also the classical result (in the special case $d=2$ relevant for quadratic forms) to the effect that $\phi_{2}(K)=4$. Write $D$ for $\operatorname{deg}(X)$. Then, with these results in hand, one may follow the argument of the proof of Theorem 4.2, mutatis mutandis, to show that $X$ possesses a $K$-rational point provided only that $\operatorname{dim}(X)$ exceeds

$$
2\left(\exp \left(8(\log D)^{2}\right)\right)^{2^{D-1}-1} \leqslant \exp \left(2^{D+2}(\log D)^{2}\right) .
$$

The conclusion of the theorem now follows.

We note that our earlier work [16, Corollaries 1.2 and 1.3] addresses the existence of rational points on certain complete intersections, over field extensions of $\mathbb{Q}_{p}$, and over purely imaginary field extensions of $\mathbb{Q}$, respectively. The proofs of these corollaries, and also the proof of [17, Theorem 10.13], use as input the main result of Skinner [14]. The correction of the latter paper embodied in [15, and improved in 3, provides a substitute for the infelicitous work of [14] that suffices to recover all of these conclusions, with one modification. Namely, the revised version of [16, Corollary 1.3] shows that when $d \in \mathbb{N}$ and $L$ is a purely imaginary field extension of $\mathbb{Q}$, then $v_{d, r}(L) \leqslant r^{2^{d-1}} e^{2^{d+1} d}$ (acquiring a factor of 2 in the exponent of $e$ relative to the original statement). We note, in particular, that when $d$ is odd and $K$ is a field extension of $\mathbb{Q}_{p}$, then the proof of [3, Theorem 1] shows that $\phi_{d}(K) \leqslant d^{2 \tau+3}$, where $d=p^{\tau} m$ with $p \nmid m$ (note in the penultimate line of that paper that $\gamma=\tau+1$ when $p \neq 2$ ). Thus, when $d$ is odd, one has $\phi_{d}(K) \leqslant e^{2 d}$, and the argument of the proof of [17, Theorem 10.13] proceeds without further modification.

Corollary 4.5. Let $L$ be an algebraic extension of $\mathbb{Q}$. Then $X$ possesses a point defined over $L$ provided only that $X$ has a point defined over all completions of $L$ at the infinite place, and in addition

$$
\operatorname{dim}(X) \geqslant \exp \left(2^{\operatorname{deg}(X)+2}(\log \operatorname{deg}(X))^{2}\right) .
$$

In particular, should this condition on $\operatorname{dim}(X)$ be satisfied, then $X$ possesses an L-rational point when $L$ is purely imaginary, and also when $\operatorname{deg}(X)$ is odd.

Proof. Frei and Madritsch [8, Theorem 1.4] show that $X$ satisfies the Hasse principle provided only that $\operatorname{dim}(X) \geqslant(\operatorname{deg}(X)-1) 2^{\operatorname{deg}(X)}-1$. But when $d \geqslant 1$, one has $\exp \left(2^{d+2}(\log d)^{2}\right)>(d-1) 2^{d}-1$, and thus the first conclusion is immediate from Theorem 4.4. When $L$ is purely imaginary, it is immediate that $X$ has a point defined over all completions of $L$ at the infinite place, since this amounts to possessing a point over $\mathbb{C}$. This confirms the second assertion of the theorem. When $\operatorname{deg}(X)$ is odd, meanwhile, it follows from Lemma 2.3(b) that $X$ is a complete intersection of hypersurfaces of odd degree. In such circumstances, it follows as before that $X$ possesses a real point, and hence the claimed result follows from the first conclusion of the theorem.

In view of [5, Remark 2.8.2], it may be of interest to restrict attention to totally real solvable extensions of $\mathbb{Q}$. Motivated by such considerations, we 
are able to derive as a special case of Corollary 4.5 a conclusion which avoids working in any extension of the groundfield whatsoever.

Corollary 4.6. Let $X \subseteq \mathbb{P}^{n}$ be a smooth and geometrically integral variety of odd degree defined over a totally real field $K$. Then $X$ possesses a point defined over $K$ provided only that $\operatorname{dim}(X) \geqslant \exp \left(2^{\operatorname{deg}(X)+2}(\log \operatorname{deg}(X))^{2}\right)$.

\section{REFERENCES}

[1] A. Bertram, L. Ein and R. Lazarsfeld, Vanishing theorems, a theorem of Severi, and the equations defining projective varieties, J. Amer. Math. Soc. 4 (1991), no. 3, 587-602.

[2] R. Brauer, A note on systems of homogeneous algebraic equations, Bull. Amer. Math. Soc. 51 (1945), 749-755.

[3] D. Brink, H. Godinho and P. H. A. Rodrigues, Simultaneous diagonal equations over p-adic fields, Acta Arith. 132 (2008), no. 4, 393-399.

[4] T. D. Browning and D. R. Heath-Brown, Forms in many variables and differing degrees, preprint; arXiv:1403.5937.

[5] M. Çiperiani and A. Wiles, Solvable points on genus one curves, Duke Math. J. 142 (2008), no. 3, 381-464.

[6] H. Davenport and D. J. Lewis, Homogeneous additive equations, Proc. Roy. Soc. Ser. A 274 (1963), 443-460.

[7] P. C. Eklof, Lefschetz's principle and local functors, Proc. Amer. Math. Soc. 37 (1973), 333-339.

[8] C. Frei and M. Madritsch, Forms of differing degrees over number fields, preprint, arXiv:1412.6419.

[9] J. Harris, Algebraic geometry. A first course, Corrected reprint of the 1992 original, Graduate Texts in Mathematics, 133, Springer-Verlag, New York, 1995.

[10] S. Lang, The theory of real places, Ann. of Math. (2) 57 (1953), 378-391.

[11] D. B. Leep and W. M. Schmidt, Systems of homogeneous equations, Invent. Math. 71 (1983), no. 3, 539-549.

[12] A. Pál, Solvable points on projective algebraic curves, Canad. J. Math. 56 (2004), no. $3,612-637$.

[13] A. Seidenberg, Comments on Lefschetz's principle, Amer. Math. Monthly 65 (1958), 685-690.

[14] C. M. Skinner, Solvability of p-adic diagonal equations, Acta Arith. 75 (1996), no. 3, 251-258.

[15] C. M. Skinner, Local solvability of diagonal equations (again), Acta Arith. 124 (2006), no. $1,73-77$.

[16] T. D. Wooley, On the local solubility of Diophantine systems, Compositio Math. 111 (1998), no. 2, 149-165.

[17] T. D. Wooley, Diophantine problems in many variables: the role of additive number theory, Topics in number theory (University Park, PA, 1997), 49-83, Math. Appl., 467, Kluwer Acad. Publ., Dordrecht, 1999.

School of Mathematics, University of Bristol, University Walk, Clifton, Bristol BS8 1TW, United Kingdom

E-mail address: matdw@bristol.ac.uk 